\title{
On-Line Marginal-Cost Pricing across Networks: Incorporating Heterogeneous Users and Stochastic Equilibria
}

\author{
by \\ Yong Zhao \\ Wilbur Smith Associates \\ 9009 Mountain Ridge Drive \\ Austin, TX 78759 \\ yzhao@wilbursmith.com \\ and \\ Kara Maria Kockelman \\ Clare Boothe Luce Associate Professor of Civil Engineering \\ The University of Texas at Austin \\ 6.9 E. Cockrell Jr. Hall \\ Austin, TX 78712-1076 \\ kkockelm@mail.utexas.edu \\ Phone: 512-471-0210 \\ FAX: 512-475-8744 \\ (Corresponding Author)
}

The following paper is a pre-print and the final publication can be found in Transportation Research 40B (5):424-435, April 2006.

Presented at the 83rd Annual Meeting of the Transportation Research Board, January 2004

\begin{abstract}
This paper discusses an on-line, trail-and-error implementation of marginal-cost pricing for networks with users whose values of travel time vary, whose demand functions are unknown, and whose route choices conform to random-utility maximization. It is an extension of Yang et al's (2004) calculations of optimal congestion tolls with homogenous travelers and shortest-path choices. The numerical example on an actual, large-scale network suggests the heuristic iterative procedure does converge in searching for optimal tolls.
\end{abstract}

Key Words: Marginal cost pricing, congestion pricing, toll roads, value of travel time 


\section{INTRODUCTION}

Road tolls based on marginal social delay costs have long been considered an economically efficient solution to highway congestion (Vickrey 1969). In most markets, goods should not be allocated beyond the point where marginal gains equal the marginal cost (MC) to furnish the good. And in certain, imperfect markets, the presence of unpriced externalities stymies this relationship. Such is the case of road use, where travelers are generally oblivious to the delays imposed on those following them during dense traffic conditions, and consider only the average travel time, or average cost (AC), they experience directly. A marginal-cost-pricing (MCP) strategy charges the user any difference between average cost and marginal costs, theoretically driving the market to a level of flow where marginal costs and benefits equate.

Knowing demand for travel across a network, one can iteratively solve for the set of prices that equate $\mathrm{MC}$ and marginal benefits (MB) on all links. In practice, however, demand functions are unknown. Li (2002) initiated and Yang et al (2004) expanded a trail-and-error implementation of MCP on a network without knowledge of demand functions but with known link performance functions, observed flows, and observed responses to pricing decisions. The procedure they propose assumes a single value of time for all users and computes the optimal prices at any demonstrated flow levels. It then relies on a diminishing fraction of the difference in optimal and current toll values, in order to adjust current tolls.

Yang et al's calculations are based on some important assumptions, including user equilibrium (UE) or shortest-path route choices and a single, known value of travel time (VOTT) for all vehicles. The UE assumption requires full information of roadway conditions (and prices) on the part of all drivers and a focus on travel time (rather than other elements of travel, such as number of stops, reliability, and route aesthetics). A more realistic network assignment assumption is believed to be stochastic user equilibrium (SUE), where each user may perceive different path costs or benefits, and random variation in route and/or traveler characteristics results in a distribution of route choices, for the same origin-destination (O-D) pair at the same time of day. In addition, travelers value their time differently, depending on the purpose of their trip and their willingness and/or ability to pay. To relax this restriction, one can segment the entire population of users into a number of groups or classes, according to their incomes and other demographic characteristics related to VOTT. For each traveler group, the users share the same the VOTT probability distributions. The distributions can be modeled as either continuous or discrete (see, for example, Cantarella and Binetti, 1998).

This paper extends Yang et al's model, algorithm and application by (1) allowing SUE network assignment, (2) recognizing group-based variations in VOTT, and (3) applying the model to a realistic, regional network. As in virtually all MCP network applications, this work assumes that MC and MB are based on travelers' own VOTTs, as evidenced through their willingness to pay (to save time). However, one can argue that some other, social VOTT applies for definition of MCs and MBs, and for selection of optimal tolls. In such situations, the optimizing-toll equations used here can be altered, to reflect the MC and MB definitions; the solution algorithms would remain the same.

The following section presents some notation and assumptions commonly adopted in SUE analysis and introduces the model for determining MCP tolls. Section 3 describes the trialand-error implementation algorithm used to calculate the optimal tolls, and Section 4 reports the results of the heuristic algorithm's application to a real, large-scale network. Conclusions and future research directions are provided in Section 5. 


\section{MCP MODEL: SUE WITH MULTIPLE USER CLASSES}

Network flow equilibria represent an interaction between congestion and travel decisions. The situation is characterized by two sets of functions: (1) performance functions for all network links, describing how travel times rise with demand; and (2) demand functions, illustrating how travel demand responds to travel-time increases (Sheffi, 1985). These demand functions are a combination of trip-making, mode choice, destination choice, route choice, and departure-time decisions.

In the stable, equilibrium condition, individual travelers cannot improve their travel times by unilaterally changing routes. This is known as Wardrop's first principle (Wardrop, 1952), and the result is a UE. Since it is unlikely that all travelers have full information about minimum travel times on every possible route and always make shortest-time route choices, the SUE equilibrium condition is a more popular principle. It is described as a network condition where no traveler can improve his/her perceived travel time by unilaterally changing routes. (Daganzo and Sheffi, 1977).

SUE assignment has been formulated as optimization (Daganzo and Sheffi, 1977), variational inequality (Bell and Iida, 1993), and fixed-point problems (Daganzo, 1983; and Cantarella, 1997). Here, the optimization formulation is followed. Daganzo (1983) proposed an SUE framework with multiple user classes, and Maher and Zhang (2000) provided a formulation and algorithms for SUE with elastic demand. Rosa and Maher (2002) extended these formulations to develop SUE with both multiple user classes and elastic demand. This paper's formulation is built on the latter three works.

Let $G(N, A)$ be a transportation network with the node set $N$ and the link set $A$, with positive monotonically increasing link performance (travel time) functions $t_{a}\left(x_{a}\right)$ of flows ${ }^{1} x_{a}$ for each link $a \in A$. The link performance function is assumed to be differentiable (with respect to flow $X_{a}$ ). Network users are segmented into $M$ classes and each class shares the same socioeconomic characteristics. Class $m$ 's demand for travel between O-D pair $r$ and $s$ at a particular time of day is denoted by $q_{m}^{r s}$, and it is assumed to be a continuously decreasing function of travel cost between that O-D pair ${ }^{2}$. The multi-user class SUE problem has an equivalent minimization formulation, which can be written as follows:

$$
\begin{array}{ll} 
& \min _{\bar{x}} Z(\vec{x})=-\sum_{r s m} q_{m}^{r s} S_{m}^{r s}(\vec{x})+\sum_{a} t_{a}\left(x_{a}\right) x_{a}-\sum_{a} \int_{0}^{x_{a}} t_{a}(w) d w \\
\text { s.t. } & x_{a}=\sum_{r s k m} f_{k m}^{r s} \delta_{a, k m}^{r s}, \forall a \\
& \sum_{k \in K^{r s}} f_{k m}^{r s}=q_{m}^{r s}, \forall r, s ; \text { and } \\
& f_{k m}^{r s} \geq 0 .
\end{array}
$$

where:

$K^{r s}$ is the set of paths between O-D pair $r$ and $s$;

$f_{k m}^{r s}$ is the flow of (demand by) class $m$ users on path $k$ between O-D pair $r$ and $s$;

$\delta_{a, k m}^{r s}=1$ if the path flow $f_{k m}^{r s}$ uses link $a$, and 0 otherwise;

$C_{k m}^{r s}$ is the travel cost of class $m$ users on path $k$ between O-D pair $r$ and $s$; and

$S_{m}^{r s}(\vec{x})=E\left[\min _{k \in K^{1 s}}\left\{C_{k m}^{r s}\right\} \mid \vec{C}^{r s}(\vec{x})\right]$ 
Note that equation (5) is the expected value of the perceived minimum travel cost. It is concave with respect to $C^{r s}(\vec{x})$ (permitting the minimization problem to have a unique solution), and its first derivative is the following:

$$
\frac{\partial S_{m}^{r s}\left(\vec{C}^{r s}\right)}{\partial C_{k m}^{r s}}=P_{k m}^{r s},
$$

where $P_{k m}^{r s}$ is the probability of class $m$ users choosing path $k$ between $r$ and $s$.

The path choice probability, $P_{k m}^{r s}$, is assumed to depend on the utility function:

$$
U_{k m}^{r s}=-\theta C_{k m}^{r s}+\varepsilon_{k m}^{r s},
$$

where $\theta$ is a behavioral parameter and $\varepsilon_{k m}^{r s}$ is a random term. If $\varepsilon_{k m}^{r s}$ follows a Gumbel distribution and is iid for all routes of interest ${ }^{3}$ (in each individual traveler's path choice decision), route choice follows a logit model. If $\varepsilon_{k m}^{r s}$ follows a normal distribution, choice confirms to a probit model. Both are valid, and, in fact, the result should hold for any concave behavioral model of route choice. In any specification, the following should hold:

$$
P_{k m}^{r s}=\operatorname{Pr}\left[\varepsilon_{k m}^{r s}-\varepsilon_{j m}^{r s} \geq-\theta C_{k m}^{r s}+-\theta C_{j m}^{r s} \forall j \in K^{r s}\right] .
$$

The VOTT dispersion among users can be modeled using continuous random variables (with a known mean value) within each class, or a fixed value per class. Without loss of generality, a single fixed-value approach (per class) is used here ${ }^{4}$.

Assuming travel cost additivity, path cost is:

$$
C_{k m}^{r s}=\sum_{a m} \operatorname{VOTT}_{m} \delta_{a, k m}^{r s} t\left(x_{a}\right)+\sum_{a m} \delta_{a, k m}^{r s} \tau_{a},
$$

where $\tau_{a}$ is the toll charged on link $a$; and $V_{\text {VOTT }}$ is the VOTT of class $m$ users.

The first derivative of the objective function with respect to the path flow yields:

$$
\frac{\partial Z(\vec{f})}{\partial f_{k m}^{r s}}=\left(-q_{m}^{r s} P_{k m}^{r s}+f_{k m}^{r s}\right) \sum_{a} \delta_{a, k m}^{r s} t_{a}^{\prime}\left(x_{a}\right),
$$

which must be zero at a stationary equilibrium point, and thus $f_{k m}^{r s}=q_{m}^{r s} P_{k m}^{r s}$, which is the SUE condition.

This SUE model can be extended to cases of variable demand between O-D pairs. If demand functions $D_{r s m}$ are nonnegative and strictly decreasing in own-path cost, then $q_{m}^{r s}=D_{r s m}\left(S_{m}^{r s}\right)$ and $S_{m}^{r s}=D_{r s m}^{-1}\left(q_{m}^{r s}\right)$.

Rosa and Maher (2002) proposed an SUE model with elastic demand that can be formulated as the following unconstrained optimization problem:

$$
\begin{aligned}
& \min _{\vec{x}, \vec{q}} Z(\vec{x}, \vec{q})=\sum_{a} t_{a}\left(x_{a}\right) x_{a}-\sum_{a} \int_{0}^{x_{a}} t_{a}(w) d w+\sum_{r s m} D_{r s m}^{-1}\left(q_{m}^{r s}\right) D_{r s m}\left(S_{m}^{r s}(\vec{x})\right) \\
& -\sum_{r s m} S_{m}^{r s}(\vec{x}) D_{r s m}\left(S_{m}^{r s}(\vec{x})\right)+\sum_{r s m} \int_{0}^{q_{m}^{r s}} D_{r s m}^{-1}(q) d q-\sum_{r s m} q_{m}^{r s} D_{r s m}^{-1}\left(q_{m}^{r s}\right) .
\end{aligned}
$$

where all variables are as defined earlier. Additionally,

$$
t_{a}\left(x_{a}\right) x_{a}=\int_{0}^{x_{a}}\left(t_{a}(w)+w t_{a}^{\prime}(w)\right) d w .
$$


Yang (1999) demonstrated, based on economic benefit maximization, that the MCP principle is still applicable in a network under SUE. As in Yang et al. (2004) and other works, the optimal tolls, $\tau_{a}^{*}$, for homogenous users, are set equal to total marginal costs as follows (assuming a single, unitary VOTT):

$$
\tau_{a}^{*}=x_{a}^{*} t_{a}^{\prime}\left(x_{a}^{*}\right),
$$

where $x_{a}^{*}$ is the optimal flow level (such that marginal cost equals marginal benefits) and $t_{a}^{\prime}$ is the derivative of travel time with respect to flow on that link.

Of course, equation (13) is not applicable when considering heterogeneous users with different VOTTs. Instead, the optimal toll is the demand (flow)-weighted average, representing the true marginal cost of an additional road user:

$$
\tau_{a}^{*}=\frac{\sum_{r s k m} f_{k}^{r s} \delta_{a, k m}^{r s} V_{\text {OOTT }} x_{a}^{*} t_{a}^{\prime}\left(x_{a}^{*}\right)}{\sum_{r s k m} f_{k}^{r s} \delta_{a, k m}^{r s}} .
$$

Yang and Huang (2004) suggested that when link flow-weighted VOTTs are used in determining an MCP link toll pattern to support a system optimum as a UE flow pattern, the results will differ when the optimum is measured in units of cost versus time. Here the cost (monetary) unit is used. The objective function in equation (11) is measured in time units and needs to be rewritten. After substituting (14) into (9) to get $C_{k m}^{r s}$ and then $S_{m}^{r s}(\vec{x})$ for the heterogeneous users conditions, the resulting multi-user SUE link flows with different VOTTs and demand patterns can be identified by solving the following unconstrained optimization problem:

$$
\begin{aligned}
& \min _{\bar{x}, \bar{q}} Z(\vec{x}, \vec{q})=\sum_{r s a k m} \operatorname{VOTT}_{m} \delta_{a, k m}^{r s} t_{a}\left(x_{a}\right) x_{a}-\sum_{r s a k m} \operatorname{VOTT}_{m} \delta_{a, k m}^{r s} \int_{0}^{x_{a}} t_{a}(w) d w+\sum_{r s m} V O T T_{m} D_{r s m}^{-1}\left(q_{m}^{r s}\right) D_{r s m}\left(S_{m}^{r s}(\vec{x})\right) \\
& -\sum_{r s m} S_{m}^{r s}(\vec{x}) D_{r s m}\left(S_{m}^{r s}(\vec{x})\right)+\sum_{r s m} \int_{0}^{q_{m}^{r s}} V O T T_{m} D_{r s m}^{-1}(q) d q-\sum_{r s m} q_{m}^{r s} V O T T_{m} D_{r s m}^{-1}\left(q_{m}^{r s}\right)
\end{aligned}
$$

Essentially, the flow solution to optimization problem (15) results in the theoretically optimal tolling situation provided by equation (14)'s tolls. This is the MCP model objective under SUE and with heterogeneous users. However, the demand functions $D(q)$ are not known in practice, complicating the solution for tolls and flows. A trial-and-error procedure for computing these given observed flows and flow responses to toll choices, based on $\mathrm{Li}$ (2002) and Yang et al. (2003), provides the remedy.

\section{TRIAL-AND-ERROR PROCEDURE}

One algorithm for solving SUE problems is the method of successive averages (MSA), which creates a weighted combination of the flow values of the current iteration and the previous iteration. The weighting factor, or step size, at each iteration $n$ is $\alpha=\frac{1}{n}$ (Sheffi, 1985); and the MSA solution method has been proven to converge to the unique solution (Sheffi and Powell, 1982). Rosa and Maher (2002) showed that the MSA applies to equation (11)'s problem and is guaranteed to converge in that application.

In the absence of demand functions $D_{r s m}, \operatorname{Li}(2002)$ proposed an iterative, trial-and-error procedure. First, an initial known/observed or desired/targeted demand $\vec{q}^{1}$ is loaded onto the 
network with a resulting flow pattern $\vec{x}^{1}$ and tolls are set to $\vec{\tau}^{1}=M \vec{C}\left(\vec{q}^{1}\right)-A \vec{C}\left(\vec{q}^{1}\right)$, where average travel cost is the sum of time and toll costs:

$A C_{a m}\left(\bar{q}^{1}\right)=\sum_{r s k} \operatorname{VOTT}_{m} \delta_{a, k m}^{r s} t\left(x_{a}^{1}\right)+\sum_{r s k} \delta_{a, k m}^{r s} \tau_{a}^{0}$, and marginal cost is the total additional travel cost imposed by the marginal user: $M \vec{C}(\vec{q}) \approx \frac{\partial A \vec{C}(\vec{q})^{T} \vec{q}}{\partial \vec{q}}$. Once $\vec{\tau}^{1}$ is imposed, the revealed traffic demand becomes $\vec{q}^{1}<\vec{q}^{15}$, suggesting that the initial tolls are too high. By adjusting the demand to a new level, for example, $\vec{q}^{1}<\vec{q}^{2}<\vec{q}^{1}$, the target toll is recalculated and the resulting demand level $\vec{q}^{2 \prime}$ is compared with $\vec{q}^{2}$. This generates an iterative procedure for optimal toll calculations.

Of course, network flows increase when demands rise, and these flows can be used as indicators of the unknown demands. Through electronic identification of road users (e.g., via simple transponders), road managers can ascertain who is using a particular link at any time and estimate their VOTTs, based on prior responses to pricing modifications. This is always done with some uncertainty, of course ${ }^{6}$. But, assuming that managers know the VOTT of those on each link at any moment in time, optimal tolls can be calculated using equation (14). Therefore, the iterative calculation can be implemented without knowing the demand function.

To solve the optimization problem (15), one needs to know the demand function and its inverse. Here, a similar approach as Li's (2002) and Yang et al's (2004) trial-and-error procedure is developed to find the optimal link flows and tolls defined by equation (14). One must solve for the SUE assignment with multiple user groups after imposing a trial set of link tolls.

Step 0. (Initialization) Set an initial set of feasible link flows $\left\{x_{a}^{0}, a \in A\right\}$. Let $n=1$.

Step 1. (Calculation of Toll) For each link a, calculate a link toll using:

$$
\tau_{a}^{*(n)}=\frac{\sum_{m} f_{k}^{r s(n)} \delta_{a, k m}^{r s} \operatorname{VOTT}_{m} x_{a}^{*(n)} t_{a}^{\prime}\left(x_{a}^{*(n)}\right)}{\sum_{m} f_{k}^{r s(n)} \delta_{a, k m}^{r s}} .
$$

Step 2. (Observing flows) Observe the revealed link flows $\left\{x_{a}^{(n)^{\prime}}, a \in A\right\}$ after imposing the tolls.

Step 3 (Convergence check). If $\frac{\left\|x^{(n)}-x^{(n)}\right\|}{\left\|x^{(n)}\right\|} \leq \phi$ (where $\phi$ is a pre-defined small value), then stop. Otherwise, proceed to the next step.

Step 4 (Link flow updates) Update link flows as follows:

$$
x_{a}^{(n+1)}=x_{a}^{(n)^{\prime}}+\alpha^{(n)}\left(x_{a}^{(n)^{\prime}}-x_{a}^{(n)}\right), a \in A,
$$

and set $n=n+1$. Go to Step 1 .

Yang et al (2004) proved that this trial-and-error procedure does converge and converges to the optimal solution, if the demand functions is a monotonically decreasing function of travel cost and are differentiable. A proof that the MCP model with SUE and heterogeneous users converges will be similar. While a fully, formal proof is not presented here, a brief discussion of three of Yang et al's (2004) propositions is helpful to understanding the similarity and sequence. First, it is easy to see that at the convergent solution to the MCP model with SUE and heterogeneous users, the corresponding flow and toll patterns are the optimal solution to the 
optimization problem (15). A multi-class SUE with elastic demand (15) and without constraints is a strictly convex problem, so its minimum is unique (Rosa and Maher, 2002). Second, by assuming that (1) demand functions are nonnegative and strictly decreasing in own-path costs and (2) link cost functions are positive and monotonically increasing with link flows, one can prove that a trial-and-error's updating procedure generates a feasible descent direction for the objective function. Finally, given these two prior prepositions and the fact that (1) the flow pattern is bounded and (2) the initial demand and flow pattern are feasible and not at the boundary, the MSA method does converge for link flows (Sheffi and Powell, 1982). Therefore, the trial-and-error iterations will converge to the optimal flow and tolling solution.

A practical explanation for the success of this rather simple trial-and-error procedure seeking optimal tolls can be summarized as follows. Assuming that there is a transportation facility operator who seeks to charge marginal-cost tolls on all road sections (i.e., links) in the network, he/she will first obtain (via observation and/or estimation) flow volumes and link users' information, including VOTTs. Then he/she charges equation (14)'s tolls, which result in higher tolls for higher flows. Since different users have different VOTTs, the overall marginal cost of additional users is a function of all current users' VOTTs, and their representation on the link. Therefore, the MCP toll is a flow-weighted average of VOTTs. After the tolls are applied, some users will shift route choices and forego trips. Overall, flows are likely to fall. The operator observes the new flow levels and users and computes a new set of "optimal” tolls, using equation (14). After a number of iterations applying and observing the results of these tolls, the operator finds that flows have stabilized, and optimal tolls are in effect. This procedure is based on several assumptions, of course. It will be hard to know the link users' different VOTTs with significant certainty, especially in the short run. Moreover, users cannot make optimal decisions without sufficient forewarning of link tolls, and travel demand is constantly shifting and always somewhat unknown, even for the same time of day on the same day of the week one week apart. Thus, this practical approach to optimal tolling may never achieve a true optimum. However, this is the situation operators are likely to be in, and recognition of heterogenous users and SUE route choice behavior certainly adds realism to Yang et al's (2004) pricing proposal.

\section{NUMERICAL EXAMPLE}

To illustrate the results of this MCP method, the approach is applied to Austin, Texas's road network (see Figure 1) with 14,491 nodes, 31,340 links, 1,074 traffic analysis zones, and two user classes. All links are subject to MCP tolls. The link performance function is based on the standard Bureau of Public Roads formula (BPR, 1964):

$$
t_{a}\left(x_{a}\right)=t_{a}^{0}\left(1+0.15\left(\frac{x_{a}}{G_{a}}\right)^{4}\right), a \in A,
$$

where $t_{a}^{0}$ is the free-flow travel time on link $a$ and $G_{a}$ is the "capacity" (which may be a levelof-service flow rate, rather than a true capacity).

Then the link tolls are calculated by:

$$
\tau_{a}^{*}=\frac{\sum_{r s k m} f_{k}^{r s} \delta_{a, k m}^{r s} V O T T_{m} 0.6 t_{a}^{0}\left(\frac{x_{a}}{G_{a}}\right)^{4}}{\sum_{r s k m} f_{k}^{r s} \delta_{a, k m}^{r s}}, a \in A .
$$


The initial O-D demand matrices, for network assignment, are borrowed from the region's planning model for morning peak hour passenger trips. $70 \%$ of the demand elements are assigned to the first user class and 30\% to the second user class. The VOTT of the two user classes $(m=1,2)$ is assumed to be $\$ 0.15$ and $\$ 0.25$ per minute (or $\$ 9$ and $\$ 12$ per hour), respectively.

In order to predict the resulting link flows, Yang et al (2004) solved an elastic demand assignment example (as in equation (15)); and, of course, this required knowledge of the demand functions. Similarly, an example application is pursued here. The demand functions are specified as the following:

$$
D_{r s m}^{(n)}=D_{r s m}^{0} \exp \left(-0.03 C_{r s}\right) \text {, for all } r, s, m,
$$

where $D_{r s m}^{0}$ is the initial demand element for user class $m$ between $r$ and $s$.

Yang et al (1994) pointed out that O-D matrix estimation from a UE (or SUE) solution is not unique. However, as long as the estimated O-D flows yield the current link flows (which are critical to toll calculations), the uniqueness of the O-D matrix is not a concern in this paper.

TransCAD (Caliper Co., 1996) was used here for the multi-class SUE trip assignment with different VOTTs. In the multi-class SUE assignment, a logit model of route choice is used (see Dial, 1971). Average perceived link costs are assumed to equal travel times multiplied by VOTTs, for each of the two user groups; and their iid Gumbel error terms are assumed to have a location parameter of 0 and scale parameter of 1 . By simulation, the link choice probabilities were calculated, then the flow assigned to the network, one user class at a time. Once the flow solution was obtained, the tolls were calculated and the O-D matrix for each use class was recomputed and re-assigned to the network, with new/updated tolls on all links.

The convergence threshold was set at $\phi=0.01$, and two different initial-demand assumptions were tested, one with an actual demand matrix and another with half of the actual demand matrix. Both cases converge using the MSA algorithm after about 50 iterations. The resulting tolls on the four selected links are shown in Table 1, and they are virtually identical (differing due to rounding errors). This suggests that the calculated MCP tolls are independent of the initial demand values.

Using the original actual demand matrix, two step sizes $(\alpha)$ were tested. The choice of $\alpha^{(n)}=\frac{1}{n}$ is equivalent to a standard MSA algorithm, while a value $\alpha=\frac{1}{2}$ serves as a check on Li's (2002) bisection algorithm. The convergence patterns are shown in Figure 2. As one can see, with $\phi=0.01$, the MSA converged at the 51st iteration. The bisection algorithm did not converge, even after 100 iterations. This confirms the numerical results of Yang et al (2004).

\section{CONCLUSIONS}

This paper expands upon Yang et al's (2004) model and algorithms to calculate optimal tolls with SUE and heterogeneous users without knowing network demand functions, but knowing VOTTs of all users. Essentially, the same trial-and-error procedure was shown to be applicable in implementing this more realistic MCP model, and a numerical example with an actual, large-scale network suggests that the trial-and-error procedure does converge when using the MSA algorithm but does not when using the bisection algorithm. This is consistent with Yang et al's (2004) results for a case of UE and homogeneous users.

This paper also discusses a proof of convergence of the heuristic, trial-and-error procedure, for an SUE application with heterogeneous users. Making standard assumptions of 
nonnegative, monotonic cost and demand functions, and uniqueness in SUE solution with elastic demand, the proof should be very similar to Yang et al's (2004). To extend both these works, it would be useful to have link costs interact with other links' flows (recognizing flow interactions at intersections, for example) and to be able to impose tolls only on selected links. In addition, the assumption of known VOTTs may not be realistic. While information linked to transponders or vehicle identification tags will make such estimates possible (based on vehicle type, route selection, time of day, and past travel choices, for example), it would be interesting to estimate such VOTTs within the context of the toll-setting algorithm, based on route choice decisions, in the presence of tolls and in real time, and it would be helpful to evaluate the suitability of solution search algorithms other than the MSA.

The MCP model with SUE and heterogeneous users provides a relatively realistic framework for studying optimal tolls, even when O-D demand functions are unknown. The trialand-error method proposed here is straightforward and likely to be of immediate use in places like Singapore and elsewhere, if the objective of roadway operators is true marginal cost pricing. This work also demonstrates the applicability of a commercially available transportation software (TransCAD) for implementing the model algorithms to a large-scale network. The results point to an attractive future for practical applications of MCP.

\section{ACKNOWLEDGEMENTS}

The authors are grateful to anonymous reviewers of this work for their comments and suggestions.

\section{ENDNOTES}

\footnotetext{
${ }^{1}$ The common term for link demand in the network equilibrium literature is flow. However, flows cannot exceed capacities, while demand loads routinely do, resulting in queuing and significant delays. Here the common term flow will be used. This will help avoid confusion with the demand variable $q$, which signifies total demand between zones, rather than link-specific demands.

${ }^{2}$ Demand for travel is assumed to depend on the origin and destination only, and the cost between them, regardless of other travel pattern costs. This assumption is standard in network models but is unfortunately unrealistic, since travelers often can (and do) substitute destinations (and even origins) in many of their travel decisions.

${ }^{3}$ In reality, route variations are unlikely to be independent, particularly when some routes overlap more than others. There has been some work on such specifications where routes are correlated, based on a logit framework (e.g., Koppelman and Wen 2000).

${ }^{4}$ Readers may refer to Cantarella and Binetti (1998) and Yang and Zhang (2002) for a more detailed discussion of VOTT distribution assumptions, and their role in the formulation.

${ }^{5}$ In reality, after imposition of tolls, some links may exhibit higher demand, due to substitution. The assumption of demand $D_{r s}$ depending only on O-D pair rs's travel times results in this reduced-demand result, and is a standard model shortcoming.

${ }^{6}$ Road users can trade toll tags/identification units among themselves, some users are new/unknown, and a user’s VOTT can change (from day to day and from one trip to the next).
} 


\section{REFERENCES}

Bureau of Public Roads (BPR) (1964). Traffic Assignment Manual. U.S. Dept. of Commerce, Urban Planning Division, Washington D.C.

Caliper Co, (1996) TransCAD User's Guide.

Cantarella, G.E. (1997) “A General Fixed-Point Approach to Multi-mode Multi-User Equilibrium Assignment with Elastic Demand.” Transportation Science 31, pp. 107-128.

Cantarella, G.E. and M. Binetti (1998) "Stochastic Equilibrium Traffic Assignment with Value of Time Distributed Among Users.” International Transactions in Operational Research, 5(6), pp. 541-553.

Daganzo, C.F. (1983) "Stochastic Network Equilibrium with Multiple Vehicle Types and Asymmetric, Indefinite Link Cost Jacobians.” Transportation Science 17, pp. 282-300.

Daganzo, C.F. and Y. Sheffi (1977) “On Stochastic Models of Traffic Assignment." Transportation Science, 11(3), pp. 253-274.

Dafermos, S.C. and F.T. Sparrow (1968) "The Traffic Assignment Problem for a General Network.” National Bureau of Standards. Journal of Researches. 73B, pp. 91-118.

Devarjan, S. (1981) “A Note on Network Equilibrium and Noncooperative Games.” Transportation Research, Part B. 15(6), pp. 421-426.

Dial, R.B. (1971) A Probabilistic Multi-Path Traffic Assignment Algorithm Which Obviates Path Enumeration. Transportation Research, 5 (2), 83-111.

Frank, M. and P. Wolfe (1956) “An Algorithm for Quadratic Programming.” Naval Research Logistics Quarterly 3 (1-2), pp. 95-110.

Koppelman, F.S., and C-H. Wen (2000) "The paired combinatorial logit model: properties, estimation and application.” Transportation Research 34B (2), pp. 75-89.

Li, M. Z. F. (2002) “The Role of Speed-Flow Relationship in Congestion Pricing Implementation with an Application to Singapore.” Transportation Research 35E, pp. 1-10.

Maher M.J. and X. Zhang (2000) "Formulation and Algorithm for the Problem of Stochastic User Equilibrium with Elastic Demand.” $8^{\text {th }}$ EURO Working Group Meeting on Transportation, Rome, September 2000.

Rosa, A. and M. Maher (2002) "Stochastic User Equilibrium Traffic Assignment with Multiple User Classes and Elastic Demand.” The Proceedings of the $13^{\text {th }}$ Mini-Euro Conference and $9^{\text {th }}$ Meeting of the Euro Working Group on Transportation, Bari, Italy.

Sheffi, Y. (1985) Urban Transportation Network, Prentice-Hall. Englewood Cliffs, NJ.

Sheffi, Y. and W. B. Powell (1982) "An Algorithm for the Equilibrium Assignment Problem with Random Link Times.” Network, 12(2), pp. 191-207.

Smith, M. J. (1979) “The Existence, Uniqueness and Stability of Traffic Equilibrium.” Transportation Research, 13B, pp.295-304.

Vickrey, W. S. (1969) “Congestion Theory and Transport Investment.” American Economic Review 59 (2), pp. 251-260. 
Wardrop J G. (1952) "Some Theoretical Aspects of Road Research." Proceedings of the Institute of Civil Engineers. (2), pp. 325-378.

Yang, H. (1999). "System Optimum, Stochastic User Equilibrium, and Optimal Link Tolls.” Transportation Science 33(4), pp. 354-360.

Yang, H. and H. Huang (2004) "The Multi-Class, Multi-Criteria Traffic Network Equilibrium and System Optimum Problem”, Transportation Research, 38B, pp.1-15.

Yang, H. and Zhang, X. (2002) “The Multi-Class Network Toll Design Problem with

Social and Spatial Equity Constraints.” Journal of Transportation Engineering, 128(5),

pp. 420-428.

Yang, H., Y. Iida, and T. Sasaki (1994) “The Equilibrium-Based Origin-Destination Matrix Estimation Problem.” Transportation Research, 28B, pp. 23-33.

Yang, H. and H.J. Huang (1998) "Principle of Marginal-Cost Pricing: How Does It Work in a General Network?” Transportation Research, 32A, pp. 45-54.

Yang, H., Q. Meng, and D-H. Lee (2004) Trial-and-Error Implementation of Marginal-Cost Pricing on Networks in the Absence of Demand Functions. Transportation Research, 38B, pp. 477-493. 


\section{LIST OF TABLES AND FIGURES}

Table 1. Calculated Tolls on Selected Links and their after 51 Iterations

Figure 1. Network Used for Numerical Example: Austin, Texas Roadways

Figure 2. Convergence of the Iterative Procedure, using Two Step-Size Rules

Table 1. Calculated Tolls on Selected Links and Their Flows after 51 Iterations

\begin{tabular}{|c|c|c|c|c|}
\hline \multirow{2}{*}{ Selected Links } & \multicolumn{2}{|c|}{ Initial Demand = Actual Demand } & \multicolumn{2}{|c|}{$\begin{array}{c}\text { Initial Demand }=0.5 \text { Actual } \\
\text { Demand }\end{array}$} \\
\cline { 2 - 5 } & Link Toll (\$) & Flow (vph) & Link Toll (\$) & Flow (vph) \\
\hline 1 (US 183 North) & 0.07 & 591 & 0.07 & 591 \\
\hline 2 (IH 35) & 0.10 & 4174 & 0.10 & 4175 \\
\hline 3 (US 290 E) & 0.15 & 2141 & 0.16 & 2140 \\
\hline 4 (Loop 1 S) & 0.08 & 3806 & 0.08 & 3807 \\
\hline
\end{tabular}




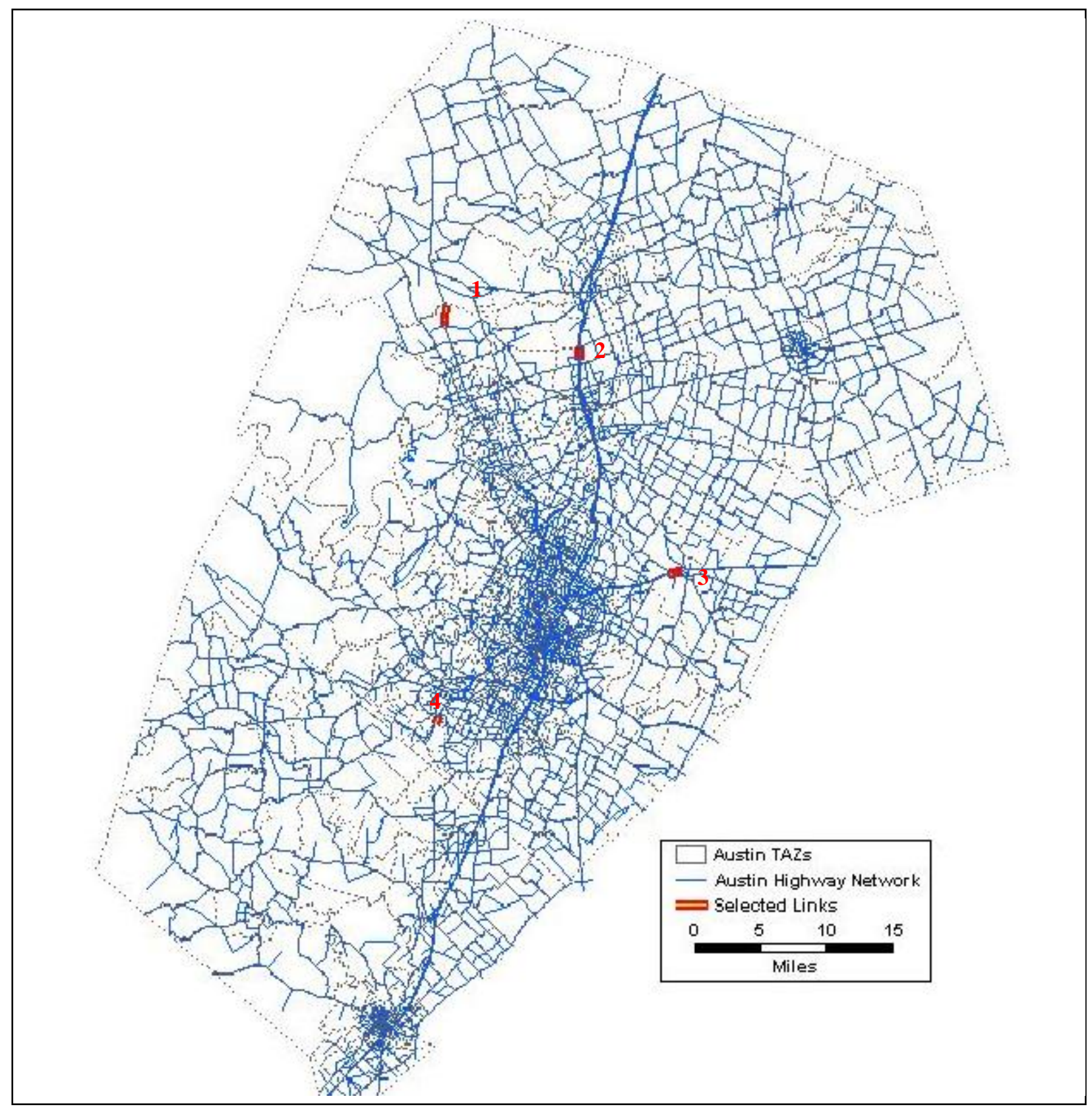

Figure 1. Network Used for Numerical Example: Austin, Texas Roadways 


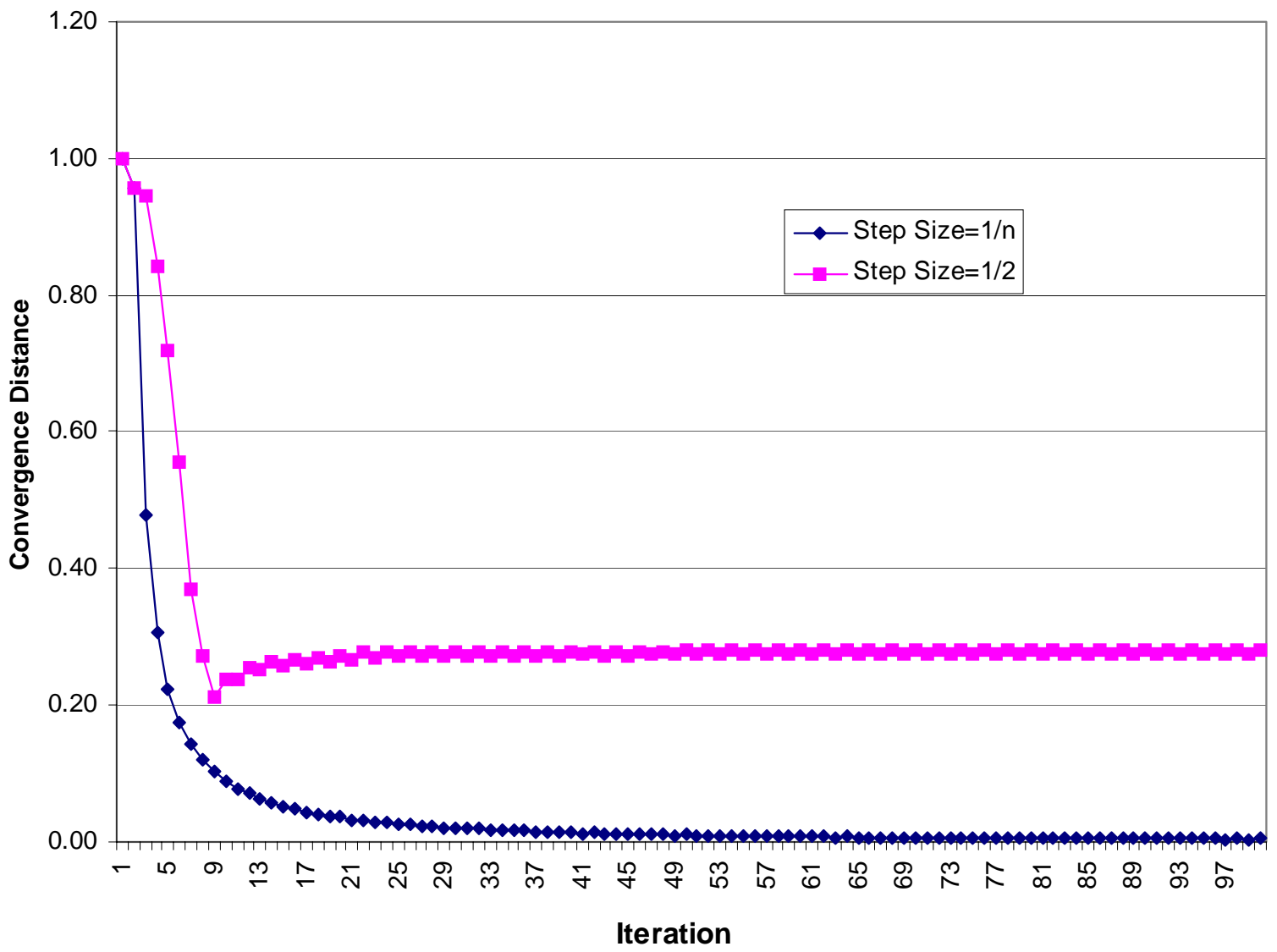

Figure 2. Convergence of the Iterative Procedure, using Two Step-Size Rules 